\title{
Las unidades didácticas en la enseñanza de las Ciencias Naturales, Educación Ambiental y Pensamiento Lógico Matemático*
}

\author{
The Iesson plans in teaching Natural \\ Sciences, Environmental Education and \\ Mathematical Logical Thinking \\ O planos de aula no ensino de \\ Ciências Naturais, Educação Ambiental \\ € Matemática raciocínio lógico
}

\author{
Omar David Álvarez Tamayo** \\ Universidad de San Buenaventura, Bogotá, Colombia
}

RECIBIDO: 16 DE AGOSTO DE 2013APROBADO: 14 DE OCTUBRE DE 2013

Resumen. El texto que se encuentra a continuación parte como un producto derivado de un estudio investigativo llevado a cabo por el

* Artículo de investigación derivado del proyecto titulado Representaciones múltiples como estrategia didáctica en la formación de conceptos en el ejercicio docente de los profesores de pregrado de la Facultad de Educación en la Universidad de San Buenaventura, Bogotá, adscrito al grupo de investigación Tendencias Actuales en Educación y Pedagogía (TAEPE), Línea de investigación en Formación y Prácticas pedagógica de la Universidad de San Buenaventura, Bogotá.

** Magister en Enseñanza de las Ciencias. Licenciado en Biología y Química. Docente de la Universidad de San Buenaventura Bogotá, Facultad de Educación. E-mail: omardavid.alvarez.tamayo@gmail.com y oalvarez@usbbog.edu.co 
autor desde lo observado en su experiencia de aula más los hallazgos obtenidos durante este proceso de investigación. En el primer apartado se desarrolla la postura de unidad didáctica a la luz de los autores Tamayo, Vasco, Suarez de La Torre, Quiceno, Castro y Giraldo (2011), la cual hace énfasis en los componentes esenciales que debe contener una unidad didáctica (ideas previas, historia y epistemología, múltiples modos semióticos y TIC, reflexión metacognitiva y evolución conceptual).

El segundo apartado gira en torno al desarrollo específico del modelo propuesto en el apartado anterior, donde se centrará la atención en la evolución conceptual partiendo de los pre-saberes de los estudiantes y cómo éstos van madurando en el estudiante. Finalmente, se presentarán las conclusiones obtenidas a lo largo de la experiencia realizada durante la observación en el aula y los aportes de la presente investigación.

Palabras claves. Unidad didáctica, evolución conceptual, enseñanzaaprendizaje (Tesauro Unesco).

Abstract. This paper, which comes from a research study carried out by the author, has been based on the researcher's class experience along with the observations captured during this investigation process. In the first chapter, the concept of didactic unit is developed and explained by different authors such as Tamayo, Vasco, Suarez de La Torre, Quiceno, Castro and Giraldo (2011). This section highlights the main components that form a didactic unit: prior knowledge, history, epistemology, multiple semiotic modes and ICT, metacognitive reflection and conceptual evolution.

The second chapter revolves around the specific development of the previously proposed model and it takes conceptual evolution as the core of the discussion by analyzing the students' prior knowledge and the way such concepts evolve. At the end, the conclusions obtained from class experiences and observations will be drawn and the contributions of the present study will be stated.

Keywords. Didactic unit, conceptual evolution, teaching-learning process (Thesaurus Unesco). 
Resumo. O texto é, então, em parte, como um subproduto de uma pesquisa realizada pelo autor da observada em sua experiência em sala de aula sobre os resultados obtidos durante o processo de investigação. Na primeira seção desenvolve o ensino a posição da unidade à luz dos autores Tamayo, Vasco, Suarez de La Torre, Quinceno, Castro e Giraldo (2011), que enfatiza os componentes essenciais que devem conter uma unidade de ensino (idéias anteriores, história e epistemologia, vários modos semióticos e TIC, reflexão metacognitiva e evolução conceitual).

A segunda seção gira em torno do desenvolvimento específico do modelo proposto na seção anterior, aonde ele irá se concentrar na base conceitual da pré- conhecimento dos alunos e como eles estão amadurecendo no aluno. Finalmente, as conclusões serão apresentadas ao longo do experimento realizado durante a observação em sala de aula e as contribuições desta pesquisa.

Palavras chave. Unidade de Aprendizagem, desenvolvimento conceitual, ensino e aprendizagem (Tesauro Unesco).

\section{Introducción}

En el presente artículo, producto derivado del proyecto titulado Representaciones múltiples como estrategia didáctica en la formación de conceptos en el ejercicio docente de los profesores de pregrado de la Facultad de Educación en la Universidad de San Buenaventura, Bogotá, se muestra la forma como se borda el diseño de Unidades Didácticas para el desarrollo de conceptos en las áreas de Ciencias Naturales, Educación Ambiental y Pensamiento Lógico Matemático en profesores y estudiantes de la Licenciatura en Educación para la Primera Infancia de la Universidad de San Buenaventura, Bogotá.

Los saberes antes nombrados (Didáctica de las Ciencias Naturales y Educación Ambiental, Pensamiento Lógico Matemático y Didáctica I, Pensamiento Lógico Matemático y Didáctica II) contribuyen a la formación científica, didáctica y humanista que el Licenciado en Educación para la Primera Infancia necesita en el ámbito de los aprendizajes que los niños experimentan en la etapa de 0 a 8 años (Facultad de Educa- 
ción, 2011); los aprendizajes desarrollados en la fase inicial conllevan a despertar la capacidad de asombro, adquisición de nociones científicas y una actitud de búsqueda de respuesta a problemas que presenta su interacción con el entorno. En este sentido la finalidad general de estos saberes es enriquecer los procesos de enseñanza-aprendizaje por medio del diseño, implementación y evaluación de situaciones que se presentan en el quehacer docente en las áreas de las Ciencias Naturales y las Matemáticas; así como aportar las estrategias, recursos y metodologías necesarias para que el futuro maestro en Primera Infancia sea capaz de aprovechar las posibilidades didácticas que le ofrece el medio.

\section{Las unidades didácticas, definición $\in$ importancia para la enseñanza y aprendizaje}

La unidad didáctica (UD) se entiende como una unidad de trabajo relativa a un proceso de enseñanza-aprendizaje, articulado y completo (MEC, 1989, citado por Gallego y Salvador, 2010, p. 303). De acuerdo con lo propuesto por los autores la UD pretende desarrollar aprendizajes significativos de una temática específica, razón por la cual es conocida como unidad relativa de trabajo.

Existen textos conformados por unidades didácticas, las UD empleadas pretenden desarrollar formación conceptual y aprendizajes significativos. No todos los textos académicos trabajan bajo el esquema de UD, se especializan en otros enfoques y diseños para impartir la información.

Muchos autores como Gallego y Salvador (2010), Tamayo, Vasco, Suarez de la Torre, Quiceno, García y Giraldo (2011), Estaire y Zanón (1990), Sánchez y Valcárcel (1993), De Pro Bueno (1999) y Tamayo (s.f), entre otros, se han dedicado a la investigación, trabajo y/o implementación de UD en diferentes áreas del conocimiento. Lo antes nombrado lleva a que se tome una postura en cuanto al trabajo realizado al diseñar e implementar UD bajo el modelo de Tamayo et al. (2011).

La propuesta por Tamayo et al. (2011), quienes definen el concepto unidad didáctica partiendo de una toma de distancia del modelo transmisionista por parte del docente y la actitud pasiva de los estudiantes, y en pro de que el alumno acoja un modelo constructivista. 
Con el modelo constructivista adoptado en el aula se pretende desarrollar en los estudiantes pensamiento científico y crítico de las problemáticas actuales en ciencias naturales, medio ambiente y matemáticas.

Tamayo et al. (2011) proponen:

$\mathrm{Al}$ ser la enseñanza una actividad que involucra distintas entidades y no una actividad de transmisión de información, vemos la necesidad de abordar la educación de las ciencias desde una perspectiva constructivista y evolutiva, en la cual se integren aspectos tales como: la historia y epistemología de los conceptos, las ideas previas de los estudiantes, la reflexión metacognitiva, los múltiples lenguajes que incluyen las TIC y el proceso de evolución conceptual como aspecto que permite una evaluación formativa, la transformación del conocimiento del pensamiento inicial y final de los docentes y de los estudiantes ( $p$ 106) (Ver Figura 1).

Este modelo de UD está conformado por cinco componentes: ideas previas, historia y epistemología de la ciencia, múltiples modos semióticos y TIC, reflexión metacognitiva, y evolución conceptual (Ver Figura 1), los cuales se discutirán posteriormente.

\section{Componentes del modelo de Unidad Didáctica}

\section{Ideas previas}

Es el primer componente que debe tener una UD bajo el modelo que se adoptó en los cursos. Se define idea previa ${ }^{1}$ como aquellos conceptos que traen los estudiantes antes de adquirir un conocimiento formal, entendido este último como el conocimiento que abarca el talento y comprensión de los conceptos científicos (Viennot, 1979; Driver, 1973; Pfundt y Duit, 1991 y Martínez, 1998, citados por Tamayo et al., 2011, p. 106). Las ideas previas de toda persona son adquiridas por múltiples fuentes como: la convivencia con otras personas, la televisión, radio, internet, leer e interactuar en un medio lleno de información, entre otras.

1 En la literatura especializada existe una gran variedad de términos empleados para hacer referencia a las ideas previas, los más conocidos son: ideas de los alumnos, concepciones erróneas, concepciones alternativas, ideas-concepciones y en inglés misconception. 
Campanario y Otero (2000), plantean que todos los estudiantes conservan un conjunto muy variado de ideas previas (preconcepciones) sobre los diferentes contenidos científicos y que la mayoría de veces las preconcepciones de los alumnos son erróneas, esto no niega que las ideas previas son muy importantes para el desarrollo de los aprendizajes significativos.

\section{Figura I.}

Modelo para la elaboración de Unidades Didácticas²

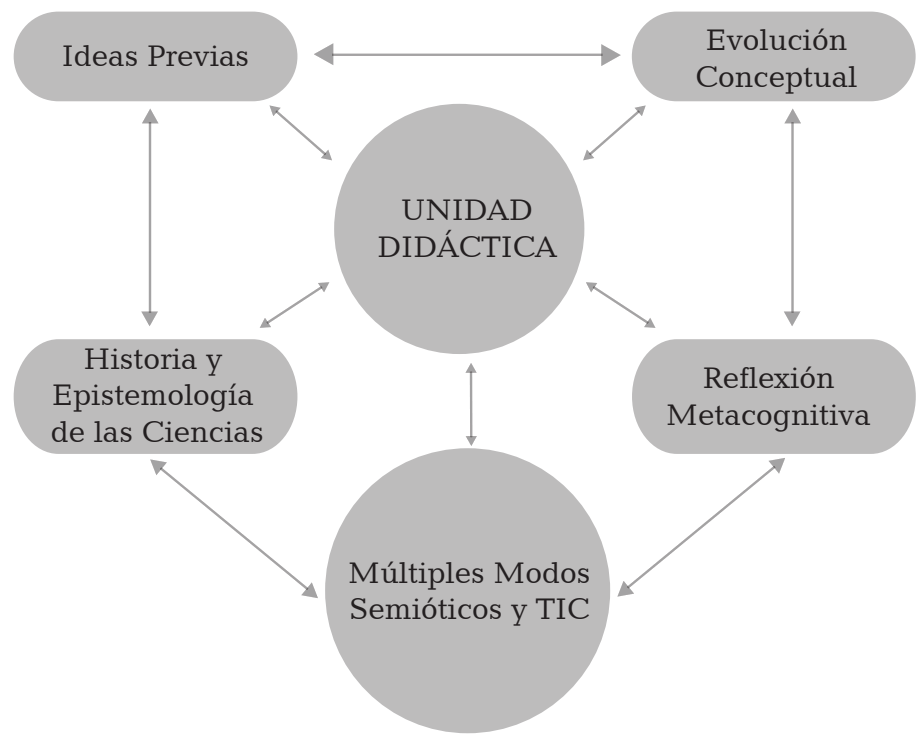

Muchas de las ideas previas están basadas en la experiencia cotidiana del estudiante; por lo general existen aspectos comunes de estas ideas previas en los alumnos sobre los conceptos científicos. Según Limón y Carretero (1997), exponen los aspectos comunes presentes en las ideas previas:

2 Modelo tomado del texto la clase multimodal y la formación y evolución de conceptos científicos a través del uso de tecnologías de la información y la comunicación (Tamayo, Vasco, Suarez de la Torre, Quiceno, Castro y Giraldo, 2011, p. 106). 
- En primer lugar, son específicas de dominio, se emplean diferentes actividades escolarizadas o desescolarizadas como las tareas para identificarlas.

- En segundo lugar, las ideas previas no se identifican fácilmente por que hacen parte del conocimiento implícito del estudiante.

- Tercero, las ideas previas son construcciones personales. A pesar de que se ha encontrado cierto grado de similitud entre las representaciones de sujetos procedentes de distintos medios culturales es necesario interpretarlas dentro del contexto individual (Driver, 1989, citado por Limón y Carretero, 1997, p. 6).

- La percepción y la experiencia de los estudiantes, adquiridas por la vida cotidiana, juegan un papel muy importante en el proceso de orientar las ideas previas.

- En cuarto lugar, no todas las ideas previas de los alumnos presentan los mismos niveles de especificidad y de generalidad, lo cual lleva a que las dificultades de comprensión que ocasionan a los estudiantes no sean de la misma importancia.

- Algunos autores como Whitey Gunstone, 1989; Pozo y Carretero, 1992; Fetherstonhaugh y Tragust, 1992 y Duit, 1994, citados por Limón y Carretero, 1997, exponen que las ideas previas son consecuentes, altamente resistentes y difíciles de modificar.

- Por último las ideas previas, tienen un nivel de coherencia y solidez variable, pueden formar parte de un modelo mental explicativo con cierta capacidad de predicción.

La importancia de la exploración de las ideas previas para los procesos de enseñanza-aprendizaje en el aula es la de identificar los diferentes niveles del conocimiento elaborado por los estudiantes a través la experiencia adquirida en la vida cotidiana.

Tamayo et al. (2011) exponen:

En el proceso de exploración de ideas previas, el docente adquiere la habilidad de agrupar las diversas de los estudiantes, de acuerdo con 
atributos similares y, en determinados casos, con modelos científicos. Estas agrupaciones o taxonomías se convierten en insumos u objetos de análisis que enriquecen la enseñanza, porque permiten hacer comparaciones con los modelos científicos. La comparación permite, según el caso, sustituir la idea previa por el conocimiento científico, actividad que da lugar a la adquisición de un conocimiento especializado (p. 107).

En coherencia con este planteamiento, la formación del conocimiento especializado se da por la constante comparación de las ideas previas de los estudiantes con los conceptos adquiridos en los cursos y que van bajo la línea de corte científico.

Queda así establecido que conocer estas ideas en los estudiantes es un ejercicio de alta importancia para el docente tal y como lo plantea Tamayo et al. (2011), dado que permite enriquecer y potenciar la planificación de toda UD por las siguientes razones:

- Al realizarse la exploración de ideas previas y con la constante interacción del docente con el estudiante se obtiene información del conocimiento común y del conocimiento científico del alumno, es por esto que se dice que las ideas previas son importantes en los procedimientos cooperativos permite que el profesor estructure las estrategias de enseñanza óptimas para el desarrollo del conocimiento científico.

- Las ideas previas permiten identificar el lenguaje empleado por el estudiante, tanto el científico como el coloquial. Identificar el lenguaje es importante en los procesos de enseñanza-aprendizaje de las ciencias, la comparación constante del lenguaje común del estudiante comparándolo el lenguaje científico permite que el docente diseñe procesos adecuados de enseñanza-aprendizaje en los que el estudiante cualificara el lenguaje, pasando de una estructura común a una científica.

- Se desarrolla una función de gran valor en la que se le da mayor importancia a la experiencia del estudiante, de esta forma se dinamizan los procesos de enseñanza dándole un giro a los modelos 
tradicionales de enseñanza (transmisionismo), pasando a uno en el que el profesor "domina los procesos de enseñanza-aprendizaje".

- Se pueden medir los grados de evolución conceptual, partiendo de las ideas previas del estudiante y la frecuente retroalimentación del docente. Se configura de esta manera una constante evaluación de las ideas del estudiante y la comparación con las ideas científicas adquiridas durante el proceso de enseñanza.

\section{Historia y epistemología de la ciencia}

Abordar este componente implica definir historia de la ciencia, epistemología y filosofía de la ciencia. Tamayo et al. (2011) proponen: "la historia de la ciencia estudia los diferentes cambios y evolución del pensamiento científico en una trayectoria espacio-temporalmente dinámica de las teorías científicas. (...) la epistemología se entiende como el estudio del conocimiento científico frente al estudio el conocimiento común" (pp. 108-109). Mellado y Carracedo (1993) plantean que desde los orígenes de la historia y la filosofía su principal problema se ha enmarcado en la fundamentación del saber, expuesto de otra forma como conocimiento científico.

Desde la filosofía clásica, exceptuando las corrientes escépticas hasta los autores de épocas modernas, reconocen dos corrientes, racionalista y empirista, desarrolladas en los siglos XVII y XVIII (Mellado y Carracedo, 1993).

El empirismo y el racionalismo son divergentes, el primero se centra en el estudio y desarrollo de la lógica, resalta el estudio de la fundamentación científica y del conocimiento; el segundo (racionalismo), destaca la importancia de la razón, en esta escuela los conceptos se desarrollan en la siguiente secuencia: primero se forman $y$, segundo, se da una fundamentación del conocimiento científico (Mellado y Carracedo, 1993). A principios del siglo XX son superadas las diferencias de estas dos escuelas filosóficas, la razón, emerge y se empieza a desarrollar un modelo constructivista, esta última escuela enfatiza en que el conocimiento es una construcción de la inteligencia humana la cual toma como punto de partida los presaberes de los estudiantes para crear estructuras nuevas en el conocimiento (Mellado y Carracedo, 1993). 
La filosofía de las ciencias juega un papel de importancia en los procesos de enseñanza-aprendizaje debido a que obliga a reflexionar constantemente en la actividad científica. Existen otras corrientes de alta relevancia en la reflexión filosófica de la actividad científica, estas son según Tamayo et al. (2011, p. 109), el positivismo lógico, la concepción heredada, la concepción historicista y el actual estructuralismo (teoría semanticista).

La pregunta consecuente es la siguiente: ¿Cuál es el papel de estos conceptos en la elaboración de Unidades Didácticas?

La respuesta es señalada por Tamayo et al. (2011), en términos de que:

- $\quad$ se ubica la temática científica que se va a enseñar en un período de tiempo específico, este período se puede relacionar con sucesos de otras disciplinas.

- $\quad$ Los desarrollos de las disciplinas son comprendidos.

- Hay que tener conocimiento de los acontecimientos históricos de la disciplina en cuestión, esto permite tener una comprensión clara de los estilos de pensamiento desarrollados en la época.

- Identificar algunos de los obstáculos que impiden el desarrollo científico y algunos de los elementos externos a la ciencia misma que catalizan su propio desarrollo, tales como: políticas educativas, políticas de desarrollo, científico, aperturas educativas a otras fronteras, entre otros (Tamayo et al., 2011, p. 110).

- Son observados los conceptos que se desarrollan o evolucionan con el tiempo y, la incidencia de la ciencia en desarrollo social.

- La historia de la ciencia incide en la evolución de la didáctica de la ciencia.

Estos elementos tienen relevancia en la intención de las unidades didácticas en la enseñanza de saberes como: Ciencias Naturales, Medio Ambiente y Pensamiento Lógico Matemático, cuyo propósito es llevar al estudiante a un aprendizaje significativo y articularlo con otras disciplinas del conocimiento. 
En la UD la epistemología y la filosofía de la ciencia hacen parte como componente integrado que permite evidenciar logros que benefician a los estudiantes y docentes, como lo justifican Tamayo et al. (2011, pp. 109-110):

- Emergen explicaciones a la luz del análisis de los fenómenos científicos.

- La interdisciplinaridad juega un papel relevante porque se da conocimiento, en la mayoría de las veces, de los productos y avances científicos, cada logro científico es fruto del trabajo colectivo de la comunidad científica.

- Las explicaciones de corte científico y las no científicas son comparadas, emergiendo explicaciones de una gama de conocimientos como: religioso, mitológico, político, y de sentido común.

- Se da la adquisición de habilidades específicas como: lenguaje especializado, desarrollo de métodos de trabajo y pericia en el empleo de instrumentos.

- Se realiza un acercamiento de los estudiantes al conocimiento científico, adquirido por la orientación del docente, trabajo con textos de rigor científico y prácticas de laboratorio, resolución de ejercicios, puestos en escena, entra otras. Se desarrolla interés por parte del estudiante en búsqueda del conocimiento.

La historia, epistemología y filosofía de la ciencia son un componente vital en la elaboración de UD, permitiendo en estudiantes y maestros desarrollar un conjunto de habilidades o beneficios que llevan a la construcción de ciencia partiendo de la importancia de los hechos científicos del pasado, la interdisciplinaridad en los saberes y la búsqueda del aprendizaje significativo.

\section{Múltiples modos semióticos y TIC}

Este concepto implica el concepto representaciones, el cual se detallara a continuación abordándolo desde las ciencias cognitivas y la didáctica

3 Tecnologías de la información y de la comunicación. 
Y, como es este empleado en la elaboración y desarrollo de Unidades Didácticas.

Duval (2004) expone tres nociones diferentes sobre representación, cada una con connotación diferente. La primera, es la de representación mental, basada en los estudios de Piaget sobre la representación del mundo en el niño; el segundo nominativo evolucionó al concepto de representación interna o computacional, que trae consigo las teorías que dan trascendencia al tratamiento ${ }^{4}$ de las representaciones; la tercera y última noción de representación es la representación semiótica, "relativa a un sistema particular de signos".

Desde la perspectiva de las ciencias cognitivas, las representaciones son consideradas como cualquier noción, signo o conjunto de símbolos que significan algo en el mundo exterior o el mundo interior (Tamayo, 2006, p. 39). Todo lo que perciben los sentidos (olfato, gusto, tacto, audición y visión) lo representa la mente (Tamayo, 2006).

Las personas pueden representar todo lo que tienen en la mente como lo que se encuentra en el medio, dicha representaciones pueden ser internas o externas (Duval, 2004; Tamayo, 2006; Diez y Caballero, 2004). Las representaciones internas se encuentran en la mente del ser humano, prácticamente se pueden considerar como tácitas, en cuanto a las representaciones externas son materializadas por medio de dibujos, gráficos, escritos, entre otros.

Tamayo (2006), plantea que las representaciones internas, mentales; se encuentra en la mente de los estudiantes, esta clase de representación permite mirar el objeto en ausencia total de significante perceptible; dentro del grupo de las representaciones internas se encuentran: creencias, nociones, fantasías, modelos mentales, conceptos, imágenes, entre otras.

Las representaciones externas son las que producen las personas o un sistema, estas representaciones son producto de emplear sistemas semióticos, lo que quiere decir que "por naturaleza son representaciones semióticas" (Duval, 2004, p. 34). Por medio de estas representaciones externas se pueden desarrollar procesos de enseñanza-aprendizaje muy

4 El tratamiento es el proceso que se le hace a la representación para que genere respuestas adaptadas. 
completos, la razón de esto es la variedad de las representaciones, pictóricas (dibujos, mapas, diagramas) y lingüísticas (notaciones simbólicas, empleo de palabras, descripciones).

El emplear múltiples representaciones externas al momento de enseñar permitirá retroalimentar los diferentes procesos conceptuales en la enseñanza de un curso, el pasar de una representación gráfica a una proposicional o viceversa llevará a que el estudiante desarrolle varios procesos cognitivos como la categorización, formación de conceptos, evolución conceptual o cambio conceptual.

Por otra parte como lo plantea Tamayo et al. (2011, p. 100):

La utilización de las TIC en los procesos de enseñanza y aprendizaje puede agregar valor a la construcción de conocimiento, en la medida que apoyan el individuo y al grupo en la creación de representaciones mentales y sociales respectivamente. Asimismo, contribuyen al desarrollo de competencias para la toma de decisiones a la solución de problemas y, a la vez, facilitan el intercambio de conocimientos y experiencias, dadas las sinergias propias de las redes de aprendizaje mediadas por tecnología (p. 100).

Antes de hablar de las nuevas tecnologías en el campo educativo primero se debe hacer un análisis reflexivo sobre los procesos de enseñanza-aprendizaje, con el propósito de comprender el uso de la tecnología en la educación (Tamayo et al., 2011), centrado en la solución de las siguientes preguntas: ¿Qué se quiere enseñar?, ¿Cómo se va a enseñar?, ¿Para quién se enseña y cómo aprende? después de dar respuesta a los interrogantes el profesor logra identificar una metodología adecuada de enseñanza-aprendizaje reflejada en apoyos tecnológicos y virtuales adecuados a los intereses del desarrollo de un aprendizaje significativo.

Se pasa, entonces, a la identificación de metodologías que incorporen la tecnología adecuada como una herramienta didáctica en los procesos de enseñanza y que potencie los aprendizajes. En búsqueda de abordar procesos de enseñanza-aprendizaje las TIC son un método que rompe los paradigmas de lo tradicional, por las siguientes razones expuestas por Tamayo et al. (2011): 
- Permiten la estructuración del conocimiento de formas diferentes gracias a las múltiples ventajas que brinda la tecnología como: multimedia, hipermedia, hipervínculos, entre otros.

- La construcción del conocimiento se puede desarrollar de una forma más sencilla gracias a que este se puede comunicar haciendo uso de los productos y análisis que realizan los autores para materializar las diferentes ideas, algunos ejemplos son los hipervínculos empleados para articular mapas mentales, mapas conceptuales, graficas, entre otros.

- Facilita la interacción con las diferentes comunidades académicas y científicas con herramientas como foros virtuales, plataformas virtuales, chats, correos electrónicos entre otros.

- $\quad$ Permite el empleo de expertos temáticos de una forma sencilla como bases de datos, bibliotecas virtuales, laboratorios virtuales, fuentes de información especializada, entre otros (p. 114).

Las TIC permiten acceder y explorar múltiples contextos y hacer que los procesos de enseñanza-aprendizaje sean innovadores y de esta forma romper paradigmas en la educación tradicional.

\section{Metacognición}

La metacognición se define como el dominio y regulación que tiene el sujeto sobre sus propios procesos cognoscitivos (Flavell, 1976, citado por Tovar, 2008, p. 3). Para Palincsar y Brown (1997) citados por Maturano, Soliveres y Macías (2002), el conocimiento metacognitivo consiste en el monitoreo activo y la regulación que se genera después de realizar actividades de procesamiento de la información. En el campo de la Didáctica de las Ciencias la metacognición es de gran importancia debido que permite la adquisición, comprensión, retención y aplicación de lo que se aprende en los cursos (Tamayo, 2009).

En el modelo de unidad didáctica presentada, la metacognición cobra importancia cuando los docentes y los estudiantes la explicitan en el aula de clase, mediante la comunicación (verbalizaciones, escritura de textos, expresión corporal, representaciones gráficas, etc.) (Tamayo et 
al., 2011, p. 116). Mateos, 2001; Romero et al., 2002; Tamayo, 2006 y Soto, 2002 citados por Tamayo (2011), dan a conocer algunos resultados de las investigaciones en las que se identifica que trae el conocimiento de los procesos cognitivos y la regulación del diseño y aplicación de la unidad didáctica:

- En primera instancia es de gran importancia facilitar los análisis que fueron aplicados en cada una de las actividades estructuradas para lograr aprendizajes en los estudiantes.

- Por medio de la metacognición se desarrollan adaptaciones favorables al medio escolar.

- La autorregulación cognitiva realizada por los estudiantes permite que estos experimenten otras formas de comunicación, ser creativos con la ayuda de múltiples lenguajes.

- La constante observación realizada por parte del profesor en el aula al implementar actividades metacognitivas le permite modificar la planeación de la enseñanza basado en las estrategias de aprendizaje de los estudiantes.

- El modelo de unidad didáctica citado para este artículo, las unidades didácticas en la enseñanza de las ciencias naturales, educación ambiental y pensamiento lógico matemático, permite hacer dos tipos de reflexión:

- Metaconceptual: establece vínculos entre los distintos componentes que conforman la unidad didáctica, lo que facilita comparar conceptos, la ubicación histórica y geográfica de estos, el estado de la ciencia y la autoevaluación del estudiante de la comprensión de los conceptos científicos.

- Metacognitiva: se enfoca en los procedimientos y las diferentes regulaciones empleadas al momento de ir adquiriendo el conocimiento.

- El ejercicio metacognitivo conlleva a desarrollar en el estudiante pensamiento crítico frente al contenido debido que le permite el 
autoconocimiento. Por otra parte permite identificar en el estudiante obstáculos lingüísticos, pedagógicos y epistemológicos.

\section{Evolución conceptual}

Como lo plantea Tamayo $(2001,2009)$, para lograr una comprensión sobre la evolución conceptual de la didáctica de las ciencias es fundamental dar respuesta a los siguientes interrogantes: ¿Cómo se produce la evolución conceptual de los estudiantes en el aula? y ¿Qué factores la favorecen?, estas son preguntas de no fácil respuesta, la razón es que se requiere de muchos campos del saber con fines de comprender lo que sucede en el aula.

El profesor debe en primera instancia desarrollar un ejercicio de observación para identificar a nivel general y particular en el aula cómo es que sus estudiantes evolucionan conceptualmente, posteriormente empieza a identificar cada uno de los factores que favorece y desfavorece dicha evolución y de esta forma cualificar su ejercicio docente llevando a que sus alumnos logren evolucionar sus conceptos.

Se han encontrado conflictos conceptuales en los estudiantes entre los conceptos iniciales y los conceptos científicos, dicho conflicto empieza a ser solucionado por el docente al incorporar en su ejercicio dentro de sus cursos la unidad didáctica, la cual por medio de sus actividades empieza a identificar satisfacción de los modelos científicos con los modelos iniciales (Tamayo et al., 2011).

Las múltiples actividades propuestas dentro de una UD permiten erradicar desde diferentes frentes los conflictos conceptuales de los estudiantes llevando a demostrar interés por parte del estudiante, una evolución conceptual y hasta llegar al desarrollo de aprendizajes significativos.

El componente evolución conceptual dentro de la UD facilita las labores del profesor y del estudiante según Tamayo (2011) por las siguientes razones:

- Permite que tanto la unidad didáctica como cada uno de sus componentes sean evaluados constantemente, logrando de esta forma conseguir e identificar la evolución conceptual alcanzada con la UD.

- Los modelos mentales tanto del profesor como de los estudiantes adquiridos por el conocimiento común y cotidiano de los fenómenos científicos son transformados. 
- Conlleva a que el estudiante logre desarrollar la capacidad de decisión para inclinarse por una teoría que le permita dar solución a las preguntas iniciales.

- Favorece el desarrollo de la creatividad con el fin de lograr una evolución conceptual por parte de los estudiantes de acuerdo con una serie de actividades propuestas por el profesor.

- La UD se nutre de los modelos mentales diferentes identificados por el maestro en el aula.

- Las fronteras existentes entre el conocimiento científico y el conocimiento cotidiano son reducidas, aterrizando la ciencia a una actividad cotidiana y sacándola del contexto de doctrina idealizada.

- El aula es transformada desde la evolución conceptual, tomando como punto de partida el aprendizaje cooperativo.

\section{Ejecución metodológica de la Unidad Didáctica}

La forma de desarrollar y ejecutar la UD tanto en Didáctica de las Ciencias Naturales y Educación Ambiental, Pensamiento Lógico Matemático y Didáctica I, y Pensamiento Lógico Matemático y Didáctica II se basa en un modelo lineal conformado por tres momentos específicos secuenciales estructurando paso a paso la evolución de los conceptos.

Los tres momentos están constituidos por actividades que permiten que el estudiante construya su propio conocimiento y a medida que van desarrollando las diferentes actividades evolucionar conceptualmente.

El primer momento consta de las siguientes actividades: en primera instancia se exploran las ideas previas, posteriormente se desarrolla una actividad que lleve al estudiante a elaborar un rastreo histórico y epistemológico del concepto estudiado, la tercera instancia es desarrollar una actividad que articule múltiples modos semióticos y el componente tecnológico con las TIC, finalizando con una actividad que lleve al estudiante a auto regularse a y a reflexionar metacognitivamente sobre el concepto estudiado (Ver Figura 2).

ITINERARIO EDUCATIVO • ISSN OIDI-Z753 • AÑO XXVII, N. ${ }^{\circ}$ G2 • JULIO - DICIEMBRE DE 2OI3 • P. II5-I35 


\section{Figura 2.}

Modelo estructural de la evolución conceptual de la UD ${ }^{5}$

\author{
Momento 1 \\ - Ideas previas \\ - Historia y \\ Epistemología del \\ conceto estudiado \\ - Múltiples modos \\ semióticos y TIC \\ - Reflexión \\ Metacognitiva
}

Momento 2
- Ideas previas
- Historia y
Epistemología del
conceto estudiado
- Múltiples modos
semióticos y TIC
- Reflexión
Metacognitiva

\section{Momento 2}

- Ideas previas

- Historia y conceto estudiado

Múltiples modos semióticos y $\mathrm{TIC}$

Metacognitiva

\author{
Momento 3 \\ - Historia y \\ Epistemología del \\ conceto estudiado \\ - Múltiples modos \\ semióticos y TIC \\ - Reflexión \\ Metacognitiva \\ - Ideas previas
}

\section{Evolución conceptual}

En un segundo momento se inicia nuevamente con la exploración de ideas previas de una forma más elaborada, con actividades similares a la exploración del primer momento con diferencia en el nivel de dificultad de la actividad (Ver Figura 2).

Por último, se inicia con actividades de un nivel de mayor dificultad de cada uno de los componentes de la UD (historia y epistemología del concepto estudiado, múltiples modos semióticos y TIC, reflexión metacognitiva) culminando con una nueva exploración de ideas previas; dicha exploración permite hacer comparación directa con la realizada en los dos momentos anteriores con el propósito de identificar en cada uno de los estudiantes si evolucionaron conceptualmente (Ver Figura 2).

A modo de conclusión, todas las unidades didácticas elaboradas bajo el modelo propuesto en el presente artículo permiten:

5 El esquema para determinar la posible evolución conceptual se centra en la exploración de ideas previas en los tres momentos de la UD, en el momento 1 y 2 las ideas previas son la primera actividad, para el momento 3 la exploración de ideas previas se hace al final, permitiendo hacer comparaciones entre cada una de estas y así determinar procesos evolutivos. 
- $\quad$ Ser diseñadas para cualquier área del conocimiento o para todos los conceptos que estas abarquen.

- Tomar múltiples rutas para lograr aprendizajes significativos, esto quiere decir que se puede elaborar unidades didácticas enfocadas en procesos metacognitivos, representacionales, basadas en las TIC o, basadas en el componente histórico y/o epistemológico de un concepto (Tamayo, et al, 2011).

- $\quad$ Que los estudiantes gradualmente construyan su propio conocimiento.

- Identificar los presaberes de los estudiantes y como estos evolucionan.

- La reconstrucción histórica y epistemológica de un concepto en un área específica del saber.

- La construcción de un concepto basado en la interdisciplinaridad de saberes.

\section{Referencias}

Campanario, J.M. y Otero, J.C. (2000). Más allá de las ideas previas como dificultades de aprendizaje: las pautas del pensamiento, las concepciones epistemológicas y las estrategias metacognitivas de los alumnos de ciencias. En Revista Enseñanza de las Ciencias, 18 (2), pp. 155-169.

De Pro Bueno, A. (1999). Planificación de unidades didácticas por los profesores: análisis de tipos de actividades de enseñanza. En Revista Enseñanza de las Ciencias, 17 (3), pp. 411-429.

Diez de Tacredi, D. y Caballero C., (2004). Representaciones externas de los conceptos biológicos de gen y cromosoma. Su aprendizaje significativo. En Revista de Investigación. No 56, pp. 91-121.

Duval, R. (2004). Semiosis y pensamiento humano. Registros semióticos y aprendizajes intelectuales. ( $2^{\mathrm{a}}$ Ed). Santiago de Cali: Merlín I.D. 
Estaire, S. y Zanón, J. (1990). El diseño de unidades didácticas en L2 mediante tareas: Principios y desarrollo. Comunicación, Lenguaje y Educación. 7 (8), pp. 55-90.

Facultad de Educación (2011). Proyecto académico pedagógico (PAP), Licenciatura en Educación para la Primera Infancia. Bogotá: Universidad de San Buenaventura.

Gallego Ortega, J.L. y Salvador Mata, F. (2010). Didáctica general. Enfoque didáctico para la globalización y la interdisciplinaridad. Cañizal (Ed.). En Didáctica general (pp. 293 - 319). Madrid: Pearson Prentice Hall.

Limón, M. y Carretero M. (1997). Las ideas previas de los alumnos. ¿Qué aporta este enfoque a la enseñanza de las ciencias? En Carretero (Ed.), Construir y enseñar las Ciencias Experimentales. (pp. 3-18). Argentina: Aique grupo Editor S.A.

Maturano, C.I., Soliveres, M.A. y Macías, A., (2002). Estrategias cognitivas y metacognitivas en la comprensión de un texto de ciencias. Revista enseñanza de las ciencias, 20 (2), pp. 415-425.

Mellado, V. y Carracedo, D., (1993). Contribuciones de la filosofía de la ciencia a la didáctica de las ciencias. Revista Enseñanza de las Ciencias, 11 (3), pp. 331-339.

Sánchez Blanco, G. y Valcárcel Pérez, M.V. (1993). Diseño de unidades didácticas en el área de ciencias naturales. Investigación y experiencias didácticas. 11 (1), pp. 33-44.

Tamayo Alzate, O.E. (S.F). Ambientes de aprendizaje: diseño de unidades didácticas para la enseñanza de las ciencias. Modulo del Diplomado en Ambientes de Aprendizaje. Universidad Autónoma de Manizales.

Tamayo Alzate, O.E. (2001). Evolución conceptual desde una perspectiva multidimensional. Aplicación al concepto respiración. Tesis de doctorado no publicada, Universidad Autónoma de Barcelona, Barcelona, España.

Tamayo Alzate, O.E. (2006). Representaciones semióticas y evolución conceptual en la enseñanza de las ciencias y las matemáticas. Revista Educación y Pedagogía. Vol. XVIII, No 45, pp. 37-49. 
Tamayo Alzate, O.E., (2009). Didáctica de las ciencias: La evolución conceptual en la enseñanza y el aprendizaje de las ciencias. Manizales: Universidad de Caldas.

Tamayo Alzate, O.E., Vasco Uribe, C.E., Suarez de la Torre, M.M., Quiceno Valencia, C.H., García Castro, L.I. y Giraldo Osorio, A.M. (2011). La clase multimodal y la formación y evolución de conceptos científicos a través del uso de tecnologías de la información y la comunicación. Manizales: Universidad Autónoma de Manizales.

Tovar Gálvez, J.C., (2008). Modelo metacognitivo como integrador de estrategias de enseñanza y estrategias de aprendizaje de las ciencias, y su relación con las competencias. Revista iberoamericana de educación, Vol 7 № 47, pp. 1-9. 